\title{
AN EXTENSION THEOREM FOR FUNCTIONS ON SEMIGROUPS
}

\author{
PAUL MILNES ${ }^{1}$
}

\begin{abstract}
For $S$ a semitopological semigroup, a continuous function on $S$ is said to be in $L M C(S)$ if its set of right translates is relatively compact in $C(S)$ for the topology of pointwise convergence on $S$. It is proved here that, if $S$ is a dense subsemigroup of a topological group $G$, then every function in $L M C(S)$ extends to a function continuous on $G$. This result generalizes earlier results that were arrived at independently by $\mathrm{A}$. T. Lau and the present author. Some corollaries of this result are also presented.
\end{abstract}

1. Definitions and main result. Let $S$ be a topological semigroup, that is, $S$ is a semigroup and a Hausdorff topological space, and the binary operation ( $s$, $t) \rightarrow s t$ from the product topological space $S \times S$ into $S$ is continuous. A function $f \in C(S)$ is said to be in $A P(S)(W A P(S))[L M C(S)]$ provided its set of right translates is relatively compact in $C(S)$ for the topology of norm convergence (weak convergence) [pointwise convergence on $S$ ], the left and right translates, $f_{s}$ and $f^{s}$, of $f \in C(S)$ by $s \in S$ being defined as usual by

$$
f_{s}(t)=f(s t) \text { and } f^{s}(t)=f(t s) \quad \text { for all } t \in S \text {, }
$$

respectively. $A P(S)$ and $W A P(S)$ are the spaces of almost periodic and weakly almost periodic functions in $C(S)$ and, if $\beta S$ is the spectrum of $C(S)$ (which is just the Stone-Čech compactification of $S$ if $S$ is completely regular), then it is not hard to show that a function $f \in C(S)$ is in $L M C(S)$ if and only if the function $s \rightarrow x\left(f_{s}\right), s \in S$, is in $C(S)$ for each $x \in \beta S$. And, finally, a function $f \in C(S)$ is called left (right) uniformly continuous if the function, $s \rightarrow f_{s}\left(s \rightarrow f^{s}\right)$, from $S$ into $C(S)$ is norm-continuous, and uniformly continuous if it is both left and right uniformly continuous. Notations for these last families of functions are $L U C(S), R U C(S)$ and $U C(S)$, respectively. The reader is referred to the references for more information about these function spaces.

We are now ready to state and prove our main result, which generalizes both Lemma 2.1 of [5] and Theorem 1 of [6] by achieving the same conclusion from weaker hypotheses. The proof is essentially that of [6], the difference being that a device of Lau is used to get around the possible absence of the identity in $S$.

THEOREM. Let $S$ be a dense subsemigroup of a topological semigroup $G$ that is

Received by the editors March 1, 1975.

AMS (MOS) subject classifications (1970). Primary 22A20, 43A60.

Key words and phrases. Topological semigroup, continuous function, almost periodic function, uniform continuity, extension.

${ }^{1}$ This research was supported in part by N.R.C. grant A7857. 
algebraically a group. Then every $f \in L M C(S)$ extends to a function in $C(G)$, i.e., $\left.C(G)\right|_{S} \supset \operatorname{LMC}(S)$.

Proof. If $S$ is a dense subsemigroup of $G$ and $r \in S$, then $T=r S r$ is a subsemigroup of $S$ that is dense in $G$; for, the map $t \rightarrow r t r$ is a homeomorphism of $G$ onto itself, since $G$ is a group and a topological semigroup. We now produce a contradiction from the assumption that there exist $s \in G$ and nets $\left\{s_{\alpha}^{\prime}\right\}$ and $\left\{t_{\beta}^{\prime}\right\}$ in $T$ such $\lim _{\alpha} s_{\alpha}^{\prime}=s=\lim _{\beta} t_{\beta}^{\prime}$ and $a=\lim _{\alpha} f\left(s_{\alpha}^{\prime}\right) \neq \lim _{\beta} f\left(t_{\beta}^{\prime}\right)=b$ for an $f \in L M C(S)$. This will complete the proof.

Let $s_{\alpha}=r^{-1} s_{\alpha}^{\prime}$ for each $\alpha$ and let $t_{\beta}=t_{\beta}^{\prime} r^{-1}$ for each $\beta$. Then $\left\{s_{\alpha}\right\} \subset S$ and $\left\{t_{\beta}\right\} \subset S$. Without loss we may assume $\lim _{\alpha} e\left(s_{\alpha}\right)=x \in \beta S$, where $e$ is the canonical continuous map of $S$ into $\beta S$; thus $x\left(f_{r}\right)=a$. Since $T$ is dense in $G$, there is a net $\left\{r_{\gamma}\right\} \subset T$ such that $\lim _{\gamma} r_{\gamma}=s^{-1} \in G$. Now consider the values $f$ along the "triple net" $\left\{t_{\beta} r r_{\gamma} r s_{\alpha}\right\} \subset S$. Since $f \in L M C(S)$, the function $t \rightarrow x\left(f_{t}\right)$ is continuous on $S$ and, since $t_{\beta} r r_{\gamma} r \rightarrow r \in S$ by continuity of multiplication, $x\left(f_{t_{\beta} r r_{r} r}\right)$ should approach $x\left(f_{r}\right)=a$. But

$$
x\left(f_{t_{\beta} r r_{\gamma} r}\right)=\lim _{\alpha} f\left(t_{\beta} r r_{\gamma} r s_{\alpha}\right)
$$

is close to $f\left(t_{\beta} r\right)=f\left(t_{\beta}^{\prime}\right)$ for all large enough $\gamma$ since $r_{\gamma} r s_{\alpha} \rightarrow e$. This implies that

$$
x\left(f_{t_{\beta} r r_{\gamma} r}\right) \rightarrow \lim _{\beta} f\left(t_{\beta} r\right)=b \neq a=x\left(f_{r}\right)
$$

as $t_{\beta} r r_{\gamma} r \rightarrow r$, the desired contradiction.

Counterexamples convince us that the hypotheses of the theorem cannot be weakened much. However, the conclusion may be too weak; we now state a conjecture which, to our knowledge, has been verified only in the case that $G$ is compact (Corollary 1, ahead).

Conjecture. In the setting of the theorem, $\left.L M C(G)\right|_{S}=L M C(S)$. (It follows directly from definitions that $\left.L M C(G)\right|_{S} \subset L M C(S)$.)

2. Corollaries to the main result. We now state a number of corollaries which generalize results of [5], [6]. They can be proved using methods of these papers and the theorem above. And, once again, counterexamples show that most hypotheses cannot be weakened much. (See the Remark below.)

COROLlary 1. If $S$ is a dense subsemigroup of a compact topological group $G$, then $\left.\operatorname{LMC}(G)\right|_{S}=\operatorname{LMC}(S)$. Hence $\operatorname{LMC}(S)=A P(S)$.

COROLlaRY 2. If $S$ is a dense subsemigroup of a topological semigroup $G$ that is algebraically a group, then $\left.A P(G)\right|_{S}=A P(S)$ and $\left.W A P(G)\right|_{S}=W A P(S)$. If, as well, $G$ is locally compact or a complete metric space, then $W A P(S)$ $\subset U C(S)$.

COROllary 3. If $S$ is a subsemigroup of a topological group $G$ and $S$ has compact closure in $G$, then $\left.L U C(G)\right|_{S}=L M C(S)$. If, as well, $G$ is locally compact, then $\left.\operatorname{WAP}(G)\right|_{S}=\operatorname{LMC}(S)$.

COROllary 4. If $S$ is a subsemigroup of a locally compact abelian group $G$ and the closure of $S$ in $G$ is a group, then $\left.A P(G)\right|_{S}=A P(S)$. 
COROllary 5. If $S$ is a subsemigroup of a locally compact group $G$ and the closure of $S$ in $G$ is an open subgroup of $G$, then $\left.W A P(G)\right|_{S}=W A P(S)$.

REMARK. It follows from a fundamental theorem of $A$. Weil on the completion of topological groups [8, p. 32] that the terms "compact group" and "locally compact group" can be weakened to "totally bounded topological group " and "topological group with a totally bounded neighbourhood", respectively, in these corollaries.

Added in proof. Dr. J. S. Pym has informed the author that the Conjecture stated above is false.

\section{REFERENCES}

1. J. F. Berglund, On extending almost periodic functions, Pacific J. Math. 33 (1970), 281-289.

2. J. F. Berglund and K. H. Hofmann, Compact semitopological semigroups and weakly almost periodic functions, Lecture Notes in Math., no. 42, Springer-Verlag, Berlin, 1967.

3. R. B. Burckel, Weakly almost periodic functions on semigroups, Gordon and Breach, New York, 1970. MR 41 \#8562.

4. E. Hewitt and K. A. Ross, Abstract harmonic analysis. Vol. I: Structure of topological groups. Integration theory, group representations, Die Grundlehren der math. Wissenschaften, Band 115, Academic Press, New York; Springer-Verlag, Berlin, 1963. MR 28 \# 158.

5. A. T. Lau, Invariant means on dense subsemigroups of topological groups, Canad. J. Math. 23 (1971), 797-801. MR 44 \#5407.

6. P. Milnes, Extension of continuous functions on topological semigroups, Pacific J. Math. 58 (1975), 553-562.

7. T. Mitchell, Topological semigroups and fixed points, Illinois J. Math. 14 (1970), 630-641. MR 42 \# 5245.

8. A. Weil, Sur les espaces à structure uniforme et sur la topologie générale, Hermann, Paris, 1937.

Department of Mathematics, University of Western Ontario, London, Ontario, CANADA 